\title{
Merging Artificial Intelligence \& Blockchain Technologies to Solve Academic Qualification Forgery Issues
}

\author{
Aisha Abdullah Ali Al Wahaibi ${ }^{a}$, Dr. Manju Jose ${ }^{b}$ \\ Post graduate scholar, MBA- IT, Middle East College, Oman \\ Faculty, Department of Computing, Middle East College, Oman
}

\begin{abstract}
This paper emphasizes on the possibility of merging Artificial Intelligence and Blockchain technologies to solve academic qualifications forgery issues in the educational sectors. Empirical data is collected through interviews with many specialist and technical people who is interested in the emerging technologies of the Fourth Industrial Revolution and focused group discussion in the field, as well as from reports in the reviewed literary articles. Scientific journals have also been accessed to analyze the paper goals and objectives. The findings are based on the conclusion suggest that emerging technologies can be integrated to become more efficient and effective in detecting fraud and forgery before it occurs. Considerable attention should be given to reducing and combating these issues because they have significant negative impacts on the economy and education. Accordingly, the study makes recommendations based on the results and areas of future research, considering the establishment of a unified and integrated system. Initially will be applied as a pilot in Sultanate of Oman, then gradually will be extended to the Gulf Cooperation Council States (GCC) and internationally particularly the affiliated and the recognized educational institutions to avoid the phenomena that affect the reputation and quality of education institutions and academic qualifications. In the conclusion considering the impacts of the proposed system in the education and economy as well in general. This research aims to investigate academic forgery cases in the world and then focuses in Oman. Furthermore, it explores the possibilities of merging technologies (Artificial Intelligence and Blockchain) to contribute in eliminating fraud before it occurs. In addition, to propose a framework that will help to find solutions based on the suggested integrated technologies. Finally, it assess the probabilities of the proposed solutions performance and its impacts in the academic sector.
\end{abstract}

Keywords: Artificial Intelligence; Blockchain; Solving Academic Qualification Forgery Issues.

\section{Introduction}

Oman's initiatives in the education sector have focused on achieving quality education, particularly in higher education. The main concerns are to maintain the level and quality of education create an appropriate universities environment and recruit qualified staff to create promising generations.

However, in the recent years, the fraud in academic qualification is consider as a critical and common issue. It has harmful impacts in many sectors including education. Thus, it is important to mention that, this issue is raising rapidly in the world, and quick intervention is required to solve this problem. Consequently, this research discuss the possibility of merging Artificial Intelligent and Blockchain technologies to solve academic qualification forgery issues.

(Mauri 2017) counterfeiting or forging is a global problem influencing a wide and variety of industries such as health, banking, Oil and gas, food, consumer products, luxury products and education as well .In 2017, the problem of fraud was considered as one of the highest operational risks to business environments. However, this paper focuses on the academic qualifications forgery issues. Where a large number of fraud in academic qualifications cases are listed in Oman, GCC countries and worldwide.

\section{The Key aspects To Study This Problem}

The education consider as one of the main pillars and hallmark of civilized society, the foundation of culture and the engine of the country's economy. There is no doubt that the education system is about preparing citizens, workers and leaders for the future. In addition, it is a key in building the upcoming generations.

\section{Background}

ISSN: 2167-1907
Academic qualifications in the education sector are important to enter the labour market and compete for jobs, promotions and career privileges. Likewise to obtain high administrative and academic positions. This competition leads some individuals to think about finding quick solutions to obtain qualifications illegally and unauthorized by joining fake universities. There are also institutions that have been criticized recently for issuing fraudulent academic qualifications with reasonable price. This result of this attitude leads to decline of educational level, a violation of academic integrity, an impact the country's economy, and unqualified outcomes from universities and colleges.

Since His Majesty Sultan Qaboos came to power in the Sultanate of Oman, he has focused on education (His Majesty in Education - University of Nizwa 2019), says: "We pay the main part of our interest in education - we strive to develop and improve, modernize, enrich and adapt the current knowledge of the world". Consistent with the Sultanate's importance to the development of human resources, integrating scientific thinking curricula, creating an educated generation that participates in the development process, and dealing with local and global changes efficiently and professionally. In addition to His Majesty's attention to the education sector, the Sultanate has established educational institutes that reach 104 universities and colleges that attract more than 46,000 postgraduates and 471,000 undergraduate students until this year (Ministry of Higher Education - Oman 2019). The increase in the number of students requires intensive efforts to get the best results in terms of providing qualified and certified academic cadres, but this has caused to increased issues of fraud in order to obtain jobs in academic positions. Accordingly, this issue has become a social responsibility and individuals supposed to contribute in order to seek solutions to eliminate this issue to occur especially in education.

It is also important to mention that, recently there have been huge media shouts and outcry through social media. Many tweets on Twitter and other social media accounts attracted attention of specialized institutions, interested individuals and society, to 


\section{Journal of Student Research}

spotlight on the seriousness of this phenomenon and its impacts and drawbacks on the economy and education.

No suspicion that, the forging of academic qualifications is a serious and sensitive unprecedented problem and it is overgrowing every day. Currently, there are many cases recorded in Oman (Ministry Of Higher Education - Oman 2019), the Ministry has counted about 1250 cases of forgery, including (108) cases of forgery for academic qualifications, (25) educational qualifications issued by fictitious institutions and (1117) false stamps during the period (1975-2018).

There are several serious incidents detected around the world; for example, (Sebugwaawo 2019) there about 143 fraudulent certificates cases are reported in the United Arab Emirates (UAE). As Life In Saudi Arabia -2019 has quoted that 900 plus engineers with fake degrees are in Saudi Arabia (Saudi Council of Engineers (SCE)).

Moreover, (Sebugwaawo 2019) pointed out that Iraqi's Parliament explains that 20,000 government employees, including senior officials in the country, have received fake educational certificates for jobs. In addition, it is important to reference that the United Arab Emirates has banned anyone found with a false certificate from working in any GCC country. In addition to the information gathered at the United States Department of Justice, 68 people from the UAE and 69 from Saudi Arabia purchased forged certificates from certificate mills in Washington. In addition, according to the United States, Justice Organization blacklisted more than 180 people from across the GCC for alleged US certificates purchased from unauthorized institutions.

In addition, last February, BBC radio found in an investigation of this matter that thousands of UK nationals have bought fake degrees from Pakistan. The source mentioned that degree buyers were consultants from the National Health Service in the UK, nurses, and contractors at the Ministry of Defence (BBC 2019).

According to the latest South African Qualifications Authority (SAQA) reports, at the end of January 2017, approximately 1,276 qualifications were identified as counterfeit and forged, of which 444 were the national qualification and 832 were considered foreign qualifications, based on the investigation done by (GARWE 2015).

(Roach 2018) In addition, Fraud in its various forms is spreading in China's academic sector, which has tarnished the reputation of Chinese research and universities.

There are a lot of cases are reported regarding the qualification forgery in various colleges and universities around Sultanate of Oman and other countries around the world

\begin{tabular}{|l|l|}
\hline Country & $\begin{array}{l}\text { The recorded number of forgery } \\
\text { cases }\end{array}$ \\
\hline Oman & 1250 cases of certification forgery \\
\hline UAA? & 143 fraudulent certificates \\
\hline KSA & 900 plus engineers with fake degrees \\
\hline Irag & 20,000 fake certificates \\
\hline OK & Thousands fake degrees from Pakistan \\
\hline South African Qualifications & 1,276 forgery qualifications \\
Authority (SEOA) & \\
\hline
\end{tabular}

Figure 1. Academic Qualification Forgery Cases in Oman $\&$ around the world.

that affects many businesses and other daily fields. The percentage These cases directly affect academic sector in Oman and other countries, (Job Fraud 2019) document forgery
Fourth Middle East College Student Research Conference, Muscat, Sultanate of Oman

consider a common problem of forged documents is about $48 \%$ are disclosed in the organizations includes 100 staff. However, Fraud and forgery are a constant challenge for competent institutions such as the Ministry of Higher Education accredited for certification, the Ministry of Manpower responsible for the recruitment of cadres as well as companies and institutions employing employees in various positions, and many other responsible bodies. Due to the relatively high level of false qualifications statistics, the rate of universities and counterfeit sites as well as universities of the inherent, so that enter into the subject by employing people who conduct these behaviours to produce such certificates. In recent years, many cases of fraudulent qualifications have emerged in the Sultanate of Oman and the world in general due to the multiplicity of methods, facilities and technological advances to introduce these transactions smoothly and accurately. This makes it difficult to detect cases of fake academic certificates. However, these situations affect Oman and other regions around the world, similarly in education. It is important to note that the incidence of fraud in academic degrees is on the rise, not to mention the vast numbers that are not registered. These statistics are a good indicator to understand the problem and its interest in detecting cases that were hidden from view with serious consequences.

\section{Emerging technologies able to solve academic forgery issues}

The differentiate nature and type of functioning of these two emerging technologies example: Artificial Intelligence and Blockchain, can be used to solve academic forgery issues. On academic forgery issues, Blockchain technology is exactly suitable for the purpose because researchers would want their work protected and secured. Verification of information and timestamping make them fundamental features of Blockchain technology, and research work may be verified and time-stamped using the technology. Moreover, the system would also allow students, and new researchers and others benefit from earlier work as well. Similarly, verification of education certificates, diplomas, and award of achievement may also be done using Blockchain where everyone in the system would be sharing authentic information. Above, it has been stated how Blockchain and AI can complete each other, and they may also solve these academic forgery issues (Casado-Vara, Prieto, \& Corchado 2018)

There were many researches and articles describing integrating two different emerging technologies to achieve certain goals. For example, in the literature review chapter, I have reviewed many cases of merging Blockchain with Internet of things to utilize decentralization feature of Blockchain to secure sensors and those devices. In addition, another use case to utilize Blockchain with Artificial Intelligence technologies to overcome each other's limitations.

As known that Blockchain needs a huge amount of computational power being not green. Artificial Intelligence can solve this issue by saving amount of consumed power if AI algorithm is used to define which node or participant has higher possibilities to solve the Blockhain algorithm. So, the reset of nodes will not try to consume additional power to solve this transaction. On the other hand, all know that AI demands special computers to run those algorithms with high end resources. Most of companies do not have such computers due to their high cost. Here Blockchain technology can utilize its decentralization feature to build AI platform in a decentralized-distributed platform (on Blockchain) which allow developers around the world to share their unutilized power to be used in AI algorithm purpose.

Academic forgeries can be solved through artificial intelligence, which can go beyond Blockchain capabilities. Artificial intelligence can use its machine intelligence to detect even if a work has been paraphrased to avoid plagiarism. It may also be used to detect if someone has stolen an idea without 


\section{Journal of Student Research}

referencing the original researcher. It is even effective in detecting forgery issues in arts education where a similar brush style can be detected to declare a painting fake. Thus, AI and Blockchain technologies can work together to solve these forgery issues. Both have to play their collaborative role to serve the purpose. However, very strong and well-developed algorithms have to be developed behind $\mathrm{AI}$ and Blockchain to enable them to achieve this purpose. The technology is already being used, but it is not efficient enough to end academic forgery. These emerging technologies have come to solve this problem efficiently. Therefore, they should be utilized (Dickson 2018).

\section{Artificial Intelligence Technology Concepts and History}

Artificial Intelligence is one of the great and universal fields of computer science and has a great scope in the future. (Press 2016) has written, "Artificial Intelligence tends to make the machine work as a human being." The algorithms enable devices to be intelligent machines and develop self-learning applications that reach to some extent the characteristics of the human mind in terms of thinking, planning, analysis, and troubleshooting to solve problems and making a decision.

As defined by Miller 2019, "Artificial Intelligence is the integration of human aptitude procedures by machines." The procedures entail learning, reasoning, and self-correction. The specific applications of Artificial Intelligence include speech recognition, machine visualization, and expert systems.

(Copeland 2019), Artificial Intelligence is simply the intelligence that is shown and demonstrated by machines as they emulate human intelligence. This definition of Artificial Intelligence shows that scientists have been trying to make machines independent enough to play their role as human beings. Keeping this objective in mind, the history of Artificial Intelligence is linked with early attempts to make machines act like human beings. (Rouse 2019), AI term was first used on August 31st, 1955, in a proposal prepared and contributed by John McCarthy and other scientists. After this proposal, a workshop was conducted one year later in 1956 in which this new field came active. Before this, there have been efforts of scientists to make machines intelligence enough to perform tasks themselves. However, the pace of this field increased after the fifth decade of the 20th century. In 1955, Herbert Simon and Allen Newell developed first AI program. The first industrial robot became operational in 1961 at the General Motors assembly line. Now, in the 21st century, this field has become a fully developed and revolutionary field (Press 2016).

In addition, (Chaudhary 2019), Artificial Intelligence enabled the system to learn text patterns in scanned images or actual documents so that algorithms are used to compare documents and find out how they are compatible with original documents even with simple differences to ensure system reliability. (Mauri 2019), high transparency and traceability of registered products that contain a product number that is easily traceable to its source in a fixed record that is difficult to update or verify until after the network participants' agreed.

(Nadella 2016), Microsoft has said that there are many basic concepts while discussing Artificial Intelligence such as NLP, ML, deep learning and neural networks.

(Wilder 2019) as Wilder mentioned in the 20th century, Artificial Intelligence techniques passed through the stages of development. Scientists have conceived an artificial neural network model similar to the neural network in the human mind that they applied to computational machines. Then they directed these applications and concepts to machine learning
Fourth Middle East College Student Research Conference, Muscat, Sultanate of Oman

primarily so that the device would identify and make predictions

\section{Blockchain Technology Concepts and History}

Blockchain is a system in which records of the transaction are kept under Bitcoin or any Cryptocurrency, and this record is shared across many computers linked with each other through a network (Kulikovskyi 2019). Blockchain is not very old technology as Stuart Haber and W. Scott Stornetta presented the idea behind it in 1991 for the first time. They offered a practical solution to time stamp the information contained in digital documents so that the information on them cannot be changed or tampered with. The system was based on the chain of blocks protected cryptographically. In 1992, Merkle trees were also added into the system so that many documents can be stored in one block to make it efficient. In 2004, computer scientist Hal Finney developed a system that can share information from person to person. The most notable development in Blockchain was introduced by the introduction of Bitcoin, which was a cash system managed electronically through a decentralized system. The technology is still evolving (Marr 2018).

Blockchain Technology and Applications | Microsoft Azure (2019) and Bandara, Ioras \& Arraiza (2018) highpoint that, Blockchain is a transparent and verifiable system that allows the sharing of value, assets, contracts and data. Therefore, it is a ledger and secure for online transactions and not relying on a centralized organization. This technology facilitates the sharing of business transactions and data and reduces the risk of waste and fraud.

(Blockchain Tutorial - Javatpoint 2019) Blockchain refers to a developing list of accounts, known as blocks, which are connected by the use of cryptography. Precisely, every block comprises a cryptographic botch of the preceding block transaction data and a timestamp. An open ledger can register operations from various parties in a secure way. It is known as Bitcoin Blockchain, Ethereum Blockchain, Smart Contracts or Digital Tokens. This paper is discussing the distributed ledgers where the list of transactions is reported and stored in different nodes in the network rather than being stored on a central organization or entity.

Based on the (Parzygnat 2019), discussed Blockchain properties that typically contain financial transactions, as is common in a peerto-peer network, use encryption and digital signatures to authenticate and enforce read / write access rights

In addition, some participants can write Blockchain content and allow some participants to read the content. It should be noted that, it relies on mechanisms that prevent historical records from being easily changed and discover when anyone tries to change the content

Blockchain is a bundle of databases and programs that are interested in ensuring that all peers over the network have the same data, listen, and agree to the proposed rules.

Also, "Traditional product tracking process can take up to seven days, while the Blockchain can call information in just 2.2 seconds. This confirms that the speed of tracking referred to in the Blockchain leads to reducing and reducing fraud" (Mauri 2019).

Vottun's Blockeducate - backed by Blockchain - pointed that, Blockchain is a system that is used to solve the problem of forging certificates in the education sector, where the network operates peer to peer and contains all digital transactions and also responsible for auditing that cannot be changed and prevent corruption (Blockchain For Education, Blockchain Academic Certificate 2019).

Refer to (Mauri 2019) the Blockchain helps to reduce supply chain fraud because of the high transparency and traceability of registered products that contain a product's barcode that is easily traceable to its source in a fixed record. Therefore, it is difficult to update or verify until after the network participants' consensus 


\section{Mechanism of the Combination}

Moreover, the integration of the two technologies results in a decentralized intelligence system. According to Pinto (2018), Artificial Intelligence is an intellectual system, whereas Blockchain is a decentralized system. In addition, Artificial Intelligence greatly relies on data from Blockchain and applies it to advance itself through machine learning. On the other hand, Blockchain is a technology that permits the stored data to be encrypted, and thus, it operates a fully protected and secured record where only approved operators can access. The integrated system protects sensitive data that means certain people, in this case, the university administration, can only access it. As a result, the assimilation of that would help the education system to set up a Blockchain founded in a manner that permits the incorporation of Artificial Intelligence schemes (Bandara, Ioras \& Arraiza 2018). Therefore, any unauthorized access to the data stored in the assimilated system would signal the university administration. In this way, the university administrators are able to deal with the issue of forgery.

Furthermore, the incorporation of both technologies enables command tracking. To be precise, Blockchain enables the tracking, comprehending and explanation of the judgments made by Artificial Intelligence. In some way, the choices made by Artificial Intelligence is hard for people to understand because it is capable of evaluating numerous variables autonomously from each other than human beings. If the decisions are documented on Blockchain, it enables them to be examined easily with the assurance that the record has not interfered with the information that has been recorded at the beginning of the inspection procedure (Schmelzer 2018). As a consequent, recording the decision-making process on the Blockchain helps maintain transparency in the process of data input and storage. Therefore, if this combination is administered in an education system, there would be some level of transparency in the manner in which the results are feed and stored in the system, a thus less academic sham.

\section{Use Cases of Integrating Both Technologies in Education Field to Avoid the Fraud}

A combination of Blockchain and Artificial Intelligence to prevent and deal with forgery in the education system can be used in different scenarios; first, in the insurance of academic transcripts. According to Schmelzer (2018), academic transcripts should be globally recognized and verifiable. In other words, all academic credentials must be verified before they are distributed to the respective students to countercheck the results to ensure that they correct. Secondly, the combination has made it possible to establish online classes where students can study, acquire course material, attempt quizzes, and exams on the platforms. As a consequent, educators can follow up on the students' academic progress, thereby avoiding falsification. Thirdly, the Sony Global Education, in partnership with IBM, established an educational policy that offers, security, and shares student's records. As a result, educators can use the files to track the academic qualification of any student involved in academic cheating. Lastly, a platform like U Port and Block-stack allows students to be recognized around the internet (Pinto 2018). Precisely, students can access apps on decentralized networks and have data transportability. Thus, anytime they $\log$ in any of the application, information about the login is relayed to the university administrative network. As a result, the administration can detect any suspicious activities that could lead to academic counterfeits.

\section{Combination AI and Blockchain}

The Artificial Intelligence and Blockchain find themselves in similar places when it comes to adoption, problem solving and fraud prevention; their combination may be able to help each other and solve some of the important concerns they face individually. Obviously, a system of Artificial Intelligence that functions as a limited human supervised network can benefit from a fixed ledger because the safety and security concerns of the network can be supported with the help of a non-porous Blockchain that has a wellestablished token system. If these technologies are taken as separate technologies, they are constrained in their applications; AI provides algorithms for analyzing the collected data and identifying specific patterns, while Blockchain will act as a necessary layer to validate data and ensure secure storage. It does not show any severe and comprehensive integration if they remain separate. (Petersson 2019) Blockchain technology also addresses problems faced by Artificial Intelligence, such as data availability, data access, and sharing with others. On the other hand, Artificial Intelligence eliminates the lack of Blockchain that which reduces the passage of decades in large calculations to ensure its validity. Thus, these techniques can work together to provide an integrated system to solve problems of forgery.

\begin{tabular}{|c|c|c|}
\hline Features & AI & Blockchain \\
\hline $\begin{array}{l}\text { Data avallability, data access, and sharing } \\
\text { (Database) }\end{array}$ & & $\checkmark$ \\
\hline Algorithms for analyzing data / caleculations & $\checkmark$ & \\
\hline Validate data and secure storage & & $\checkmark$ \\
\hline Supervised network & $\checkmark$ & \\
\hline Identilying specific patterns & $\checkmark$ & \\
\hline Ablility to simplity worklow & $\checkmark$ & \\
\hline Reduce human emors & $\checkmark$ & \\
\hline Accelerate the detection of fraud and change & $\checkmark$ & \\
\hline Enbling devices to communicate directly & & $\checkmark$ \\
\hline Ledger record & & $\checkmark$ \\
\hline Basis for applications involving transactions and interactions & & $\checkmark$ \\
\hline Tracking derices & & $\checkmark$ \\
\hline Provide better blling and coding methods & $\checkmark$ & \\
\hline
\end{tabular}

Table 1. Comparison between AI \& Blockchain features

Results:

\section{Traditional Ways to Spot the Fake Certification}

Refer to (Academic Qualification Fraud: How To Spot A Fake CV And What To Do 2019), Degrees of fraud vary with regard to academic qualifications and its credibility, and there are quick tests that individuals can take to determine a false qualification. A visitor or a university student can reveal what the university is and what it is really about through Google search and make sure that it is correct even though there is a website with all the details. In addition, by checking, the address of the university and detecting typos contained in the certificate or its content. Besides the absence of gold seals in the real certificates and the absence of a threedimensional image or watermark in the certificate and the presence 


\section{Journal of Student Research}

of an electronic signature in the certificate and all these things would reveal to people the validity of the certificate and the validity.

\section{The Proposed Solution}

The proposed solution is divided into two main parts. The first part is the distributed ledger based on Blockchain. It is a distributed network contains participants around the world. They are responsible to add any qualification, certification, work experience and grade into the block as electronic transaction with a unique hash. Each participants (accreditation authority, universities, educational institutions, medical training centers), have a privilege to read and write into the Block-chain system. They have the right to add a new certification into the network (write) or validating an existing qualification for the others (read). By this part of the solution can reduce the number of fake certificates or academic degrees.

The second part of the solution's job, based on Artificial Intelligence (AI) algorithm to decide which candidates are better for the vacancy based on historical data that can be gathered from the network. In addition, it has a connection to third party feeds cloud which contains most of blacklisted institutions, universities, and academic centers. With this part, it can exclude candidates those studied in different universities from the world (which is not part of the Blockchain network). Then, it will provide a recommendation for the entity to help choosing the right candidate

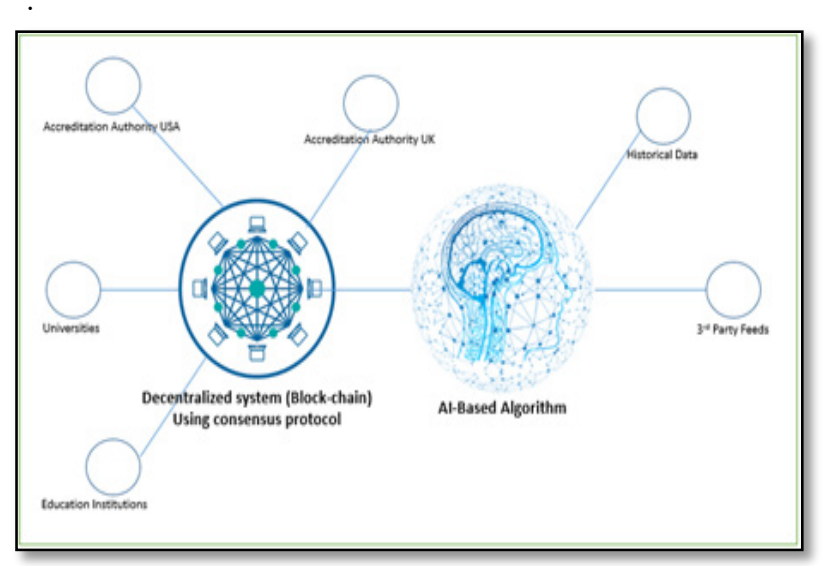

Figure 2. The proposed System by integrating AI based algorithm with distributed ledger system based on Blockchain

\section{The Proposed System Process}

The below figure (Figure 3) illustrates the following steps to be followed by the proposed system to examine and monitor the validity of job seeker certificates before the recruitment system and hiring the candidate:

Step 1: The University issues the certificate as a digital transaction with hash value

Step 2: The transaction will be broadcasted in a decentralized network

Step 3: If job seeker wants apply for a job, the entity will request to validate his qualification through the proposed system

Step 4: If the hash is matched then the qualification is valid
Fourth Middle East College Student Research Conference, Muscat, Sultanate of Oman

Step 5: Otherwise, will send these information to the AI algorithm which has international feeds collected about blacklisted universities and previous data to have a good decision

Step 6: In addition the AI system will recommend which candidate is the best for the vacancy based on the Blockchain validation and machine learning analysis for a huge amount of data.

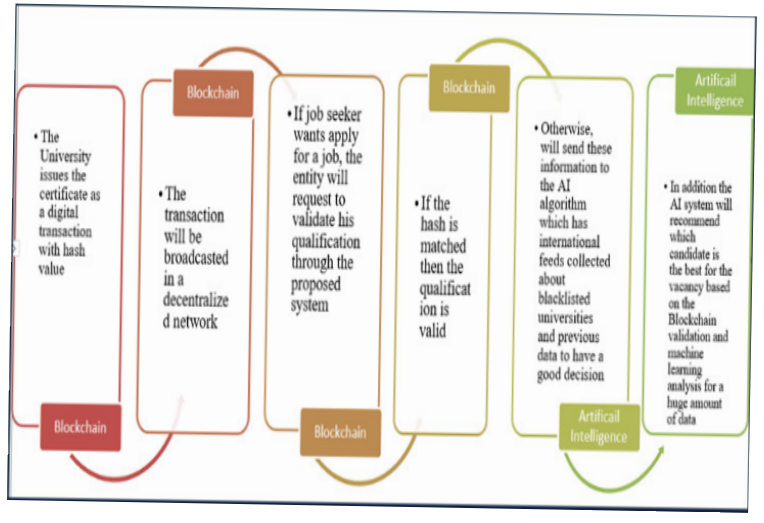

Figure 3. The Process of Proposed System

\section{The Expected Impacts of the Proposed System}

By introducing new systems and techniques in the educational sector in order to combat fraud in academic qualifications will gradually eliminate this problem. This hopefully will lead to fruitful results in the academic and the employment sectors. The following points represents the view and opinions of the interviewees about the proposed system impacts

In Education system can change for the better by:

$\square$ The high level of competencies and educational cadres will lead to better outputs

$\square$ Reducing and combating counterfeiting in the higher educational institutions qualifications deters students from purchasing false certificates

$\square$ Improving and developing the public and private education institutions

$\square$ Increasing the efficiency of education by attracting competent teachers in the academic and developmental sense

In Employment sector:

$\square$ Raise credibility in work and not to exploit academic and administrative positions to provide certificates to those who do not deserve it staff

$\square$ Ensures the presence of capable and qualified scientific

$\square$ Having job opportunities for those with real and distinctive qualifications

$\square$ Technology of them will solve the limitation of the other one. As well, there is a description of how this proposal will solve the forgery issue in academia.

\section{Conclusion and Recommendation}

This paper is built on the assumption that the interaction potential of these two technologies, to be tested and employed to solve specific issues. Based on previous similar studies and literature reviews, it shows that this experience will provide a great solution to this matter.

Due to the nature of my study field (Master of Business Administration-IT), I am not required to conduct this experiment and so, this will be part of future improvement. A detailed process 


\section{Journal of Student Research}

and steps were shared with Blockchain Solutions \& Services Company (Government Company) to provide a test VM to start implementing my project and to support necessary technical support. Especially in the area of Blockchain. The company wants to achieve this integration as it already has worked to integrate IoT with Blockchain (BSS, 2019).

\section{Conclusion and Remarks}

This paper provides a simple overview of the perceptions of the application of the fraud detection system, qualifications and academic certificates. It is imperative that the concerned parties agree to resolve this issue, adopt it, and begin implementation so that it is not only a system that has been promoted but is implemented on the ground as a unified and integrated system of educational actors and operational organizations. Based on the study of academic qualifications forgery cases around the world I understand that the impacts are almost similar. The capabilities of emerging technologies to prevent fraud in different field. However, the combined two technologies with the features listed will improve the effectiveness of their performance and functionality to solve such cases.

\section{Summary}

This paper believes that academic fraud is a controllable crime in prospective education. This research included many aspects that contributed to the understanding of the issue addressed in the research, study and discussion of these ranges to find a possible solution to solve the problem of formulating academic qualifications through the integration of artificial intelligence techniques and Blockchain. The research in Chapter 1 includes a comprehensive introduction to the research, identify the objectives addressed, and discuss the questions mentioned. The research discussed the importance of the issue and seriousness. In addition, research outputs and chapters to be followed are explained. Subsequently, various previous studies were reviewed by the researcher to reach the research objectives. The increasing detection of fraud and recorded statistics in the world is a confirmation of the interest and follow-up of the parties concerned to eliminate and reduce this problem. There are also many negative effects on the economy and education in terms of the quality of outputs and the employment of unreal competencies in administrative and academic positions. Furthermore, one of the objectives of this research is to solve the problem in terms of using emerging technologies to distinguish the reliability and validity of academic qualifications to avoid problems arising from this problem. Then discuss the possibilities of the two techniques and the possibility of combining them to reach the desired goal. Therefore, combining these two things has many benefits, including, helps Blockchain in tracking, explaining and understanding the decisions made by AI. Moreover, artificial intelligence adapts to the chains of masses more than humans; therefore, they work well together. Finally, it enables the verification of an individual's education records quickly and reasonably. As a result, the combination of the two is required to expose issues of educational qualification fraud. And it effectiveness of incorporating proposed techniques for detecting fraud before it occurs. In addition, the proposed solution or framework and process system for testing are explained in future research. Finally, the conclusion and recommendation is to try the proposed solution and apply it on
Fourth Middle East College Student Research Conference, Muscat, Sultanate of Oman

the ground to combat this problem.

\section{Acknowledgment}

Foremost, I acutely would like to express my sincere thankfulness to Dr. Manju's advisers for the continued support of my Masters and my research, for her patience, motivation, enthusiasm and knowledge. Her guidance helped me all the time in researching and writing this dissertation. I did not imagine that I would have a better adviser to study for a master's degree.

Besides my advisor, I would like to thank the teaching staff and administrators at Middle East College for their continued cooperation and constructive comments that added a lot of value to the dissertation.Last but not least, I would like to spread my thanks and gratitude to all people who support and enhance my capabilities in somehow.

\section{References}

900 ENGINEERS CAUGHT IN SAUDI ARABIA DUE TO FAKE DEGREES - LIFE IN SAUDI ARABIA (2019) AVAILABLE FROM $<$ HTTPS://LIFEINSAUDIARABIA.NET/BLOG/2019 /03/12/900-ENGINEERS-CAUGHT-IN-SAUDIARABIA-DUE-TO-FAKE-DEGREES/> $\quad\left[\begin{array}{ll}14 & \text { MAY }\end{array}\right.$ 2019]

Academic Qualification Fraud: How To Spot A Fake CV And What To Do (2019) available from $<$ https://www.marisit.co.za/how-to-spot-a-fake-cvwhat-to-do/> [26 June 2019]

Anwar, H. (2019) 6 Key Blockchain Features You Need To Know About! [online] available from $<$ https://101blockchains.com/introduction-toblockchain-features/\#prettyPhoto/0/> [1 April 2019]

Anwar, H., 2018. 101 Blockchains. [Online] Available at: https://101blockchains.com/introduction-toblockchain-features/ [Accessed 24 April 2019].

Artificial Intelligence - What It Is And Why It Matters (2019) available from <Goodnight, J. (2019) Artificial Intelligence - What It Is And Why It Matters [online] available from $<$ https://www.sas.com/en_us/insights/analytics/whatis-artificial-intelligence.html $>[10$ July 2019] $>[25$ June 2019]

Artificial Intelligence Advantages - Bing Images (2019) available from

$<$ https://www.bing.com/images/search?q=Artificail + in telligence+advantages \&go $=$ Search \&qs $=$ ds \& form $=\mathrm{QBI}$ R\&first $=1 \& \mathrm{cw}=1129 \& \mathrm{ch}=403>$ [7 July 2019]

Bandara, I.B., Ioras, F. and Arraiza, M.P., 2018. The emerging trend of blockchain for validating degree apprenticeship certification in cybersecurity education.

Blockchain Technology And Applications | Microsoft Azure (2019) available from $<$ https://azure.microsoft.com/enus/solutions/blockchain/> [10 July 2019]

Blockchain Tutorial - Javatpoint (2019) available from $<$ https://www.javatpoint.com/blockchain-tutorial $>$ [20 June 2019]

Bond, F., Amati, F. and Blousson, G. (2019) available from $<$ https://www.forbes.com/sites/forbestechcouncil/2018 /10/09/next-> [11 June 2019]

Chaudhary, M. (2019) Signature Forgery Detection Using AI Accubits Blog [online] available from $<$ https://blog.accubits.com/signature-forgery-detectionusing-ai/> [7 May 2019] 


\section{Journal of Student Research}

Chiyevo, E., 2015. Qualification, Award and Recognition Fraud in Higher Education in Zimbabwe. Journal of Studies in Education, 5(2), pp. 119-130.

Copeland, B. (2019) Artificial Intelligence | Definition, Examples, And Applications [online] available from

$<$ https://www.britannica.com/technology/artificialintelligence $>$ [28 May 2019]

Feedzai. (2015). Machine Learning and AI for Fraud Prevention: A Primer - Feedzai. [online] Available at: https://feedzai.com/resources/machine-learningai-fraud-prevention-primer/ [Accessed 24 Aug. 2019].

GARWE, E. (2015) "Qualification, Award And Recognition Fraud In Higher Education In Zimbabwe". Journal Of Studies In Education 5 (2), 119

Higher Education Ministry Detects More Than 1,200 Fake Certificates (2019) available from $<$ https://timesofoman.com/article/540700> [22 April 2019]

His Majesty On Education - University Of Nizwa (2019) available from $<$ https://www.unizwa.edu.om/index.php?contentid $=3$ \&lang=en $>$ [20 April 2019]

How AI Recruiting Technology Is Changing The Whole Industry? - Surecert (2018) available from $<$ https://www.surecert.com/blog/ai-recruitingtechnology-is-changing-the-whole-industry/> [24 March 2019]

Kulikovskyi, A. (2019) "BLOCKCHAIN AS A COMPONENT OF INFORMATION SECURITY". Cybersecurity: Education Science Technique (4)

Machine Learning And AI For Fraud Prevention: A Primer Feedzai (2018) available from $<$ https://feedzai.com/resources/machine-learningai-fraud-prevention-primer/> [19 May 2019]

Mackey, T. K., Kuo, T. T., Gummadi, B., Clauson, K. A., Church, G., Grishin, D., \& Palombini, M. (2019). 'Fit-for-purpose?'-challenges and opportunities for applications of blockchain technology in the future of healthcare. BMC medicine, 17(1), 68 .

Mar, B. (2018). Artificial Intelligence And Blockchain: 3 Major Benefits Of Combining These Two MegaTrends. Retrieved from https://www.forbes.com/sites/bernardmarr/2018/03 /02/artificial-intelligence-and-blockchain-3-majorbenefits-of-combining-these-two-megatrends/\#32bf32fe4b44

Mauri, R. (2019) Blockchain For Fraud Prevention: Industry Use Cases - Blockchain Pulse: IBM Blockchain Blog [online] available from $<$ https://www.ibm.com/blogs/blockchain/2017/07/ blockchain-for-fraud-prevention-industry-usecases/> [24 May 2019]

Miller, T. (2019) "Explanation In Artificial Intelligence: Insights From The Social Sciences". Artificial Intelligence 267, 1-38

Ministry Of Higher Education - Oman : (2019) available from $<$ https://www.mohe.gov.om/InnerPage.aspx?ID=5 3ac2db6-bf60-4958-b928-

74add9e 05ce5\&culture $=$ en $>$ [16 April 2019]

News, A. (2019) Tweet Chat: Impact Of Academic Fraud On Higher Education [online] available from $<$ https://www.asicuk.com/news/tweet-chat-impact-
Fourth Middle East College Student Research Conference, Muscat, Sultanate of Oman

Palmer, G. (2015) Tracking Axact'S Websites [online] available from

$<$ https://www.nytimes.com/2015/05/17/world/asia/trac king-axacts-websites.html> [15 May 2019]

Petersson, D. (2019) Blockchain And AI Combined Solve Problems Inherent In Each [online] available from $<$ https://searchcio.techtarget.com/feature/Blockchainand-AI-combined-solve-problems-inherent-in-each $>[8$ May 2019]

Pinto, R. (2018). Council Post: Next Steps In The Integration Of Artificial Intelligence And The Blockchain. [online] Forbes.com. Available at: https://www.forbes.com/sites/forbestechcouncil/2018/1 0/09/next-steps-in-the-integration-of-artificialintelligence-and-the-blockchain/\#1971ae513273 [Accessed 24 Jun. 2019].

Press, G. (2016). A Very Short History Of Artificial Intelligence (AI). Retrieved from https://www.forbes.com/sites/gilpress/2016/12/30/avery-short-history-of-artificial-intelligenceai/\#61db29f06fba

Procedures For Investigating Academic Fraud (2019) available from $<$ https://www.ncbi.nlm.nih.gov/books/NBK236197/> [23 June 2019]

Research Guides: Library Research Guide For The History Of Science: Introduction: What Is A Secondary Source? (2019) available from $<$ https://guides.library.harvard.edu/HistSciInfo/second ary> [27 June 2019]

Sarda, P., Chowdhury, M., Colman, A., Kabir, M. and Han, J. (2018) "Blockchain For Fraud Prevention: A WorkHistory Fraud Prevention System". 2018 17Th IEEE International Conference On Trust, Security And Privacy In Computing And Communications/ 12Th IEEE International Conference On Big Data Science And Engineering (Trustcom/Bigdatase)

Schmelzer, R. (2018). Combination of blockchain and AI makes models more transparent. [online] SearchEnterpriseAI. Available https://searchenterpriseai.techtarget.com/feature/Combi nation-of-blockchain-and-AI-makes-models-moretransparent [Accessed 24 Jun. 2019].

Tough Measures And Penalties To Check Fake Certificate Cases In Oman (2018) available from $<$ https://timesofoman.com/article/535606> [7 May 2019]

Trines, S. (2019) Academic Fraud, Corruption, And Implications For Credential Assessment [online] available from $<$ https://wenr.wes.org/2017/12/academic-fraudcorruption-and-implications-for-credentialassessment $>$ [19 April 2019]

Writer, G. (2019) If AI And Blockchain Converge, They Could Change Each Other And The World [online] available from <https://www.iotforall.com/ai-and-blockchainconverge-could-change-world/> [12 May 2019] 